\title{
Propagators for the time-dependent Kohn-Sham equations
}

\author{
Alberto Castroa) \\ Departamento de Física Teórica, Universidad de Valladolid, Valladolid, Spain and Donostia International \\ Physics Center (DIPC), 20018 San Sebastián, Spain \\ Miguel A. L. Marques ${ }^{\text {) }}$ \\ Institut für Theoretische Physik, Freie Universität Berlin, Arnimallee 14, D-14195 Berlin, Germany \\ Angel Rubio ${ }^{\text {c) }}$ \\ Departamento de Física de Materiales, Facultad de Químicas, UPV/EHU, and Centro Mixto CSIC-UPV/ \\ EHU, 20018 San Sebastián, Spain and Donostia International Physics Center (DIPC), 20018 San \\ Sebastián, Spain
}

(Received 7 April 2004; accepted 28 May 2004)

\begin{abstract}
In this paper we address the problem of the numerical integration of the time-dependent Schrödinger equation $i \partial_{t} \varphi=\hat{H} \varphi$. In particular, we are concerned with the important case where $\hat{H}$ is the self-consistent Kohn-Sham Hamiltonian that stems from time-dependent functional theory. As the Kohn-Sham potential depends parametrically on the time-dependent density, $\hat{H}$ is in general time dependent, even in the absence of an external time-dependent field. The present analysis also holds for the description of the excited state dynamics of a many-electron system under the influence of arbitrary external time-dependent electromagnetic fields. Our discussion is separated in two parts: (i) First, we look at several algorithms to approximate $\exp (\hat{A})$, where $\hat{A}$ is a time-independent operator [e.g., $\hat{A}=-i \Delta t \hat{H}(\tau)$ for some given time $\tau$ ]. In particular, polynomial expansions, projection in Krylov subspaces, and split-operator methods are investigated. (ii) We then discuss different approximations for the time-evolution operator, such as the midpoint and implicit rules, and Magnus expansions. Split-operator techniques can also be modified to approximate the full time-dependent propagator. As the Hamiltonian is time dependent, problem (ii) is not equivalent to (i). All these techniques have been implemented and tested in our computer code OCTOPUS, but can be of general use in other frameworks and implementations. (C) 2004 American Institute of Physics.
\end{abstract}

[DOI: $10.1063 / 1.1774980]$

\section{INTRODUCTION}

One of the most famous equations in physics is the ubiquitous Schrödinger equation, which is at the heart of quantum mechanics. In its time-dependent form, this equation is normally written as (we use atomic units $e^{2}=\hbar=m=1$ hereafter)

$$
i \frac{\partial \varphi}{\partial t}(t)=\hat{H}(t) \varphi(t)
$$

and describes the evolution of a wave function $\varphi$ in a system defined by the Hamiltonian operator $\hat{H}$. In the simplest case, the Hamiltonian is composed of two terms, one of kinetic origin and another describing the interaction of the particle with a local potential:

$$
\hat{H}(t)=\hat{T}+\hat{V}(t)=-\frac{\nabla^{2}}{2}+v(\mathbf{r}, t) .
$$

(In some special cases, the external potential may contain nonlocal contributions, e.g., nonlocal pseudopotentials.) This Hamiltonian is nonbounded and, in most cases, Hermitian.

\footnotetext{
${ }^{a)}$ Electronic mail: alberto.castro@tddft.org

b)Electronic mail: marques@tddft.org

${ }^{c)}$ Electronic mail: arubio@sc.ehu.es
}

Besides appropriate boundary conditions, without which the kinetic term is not properly defined, the time-dependent Schrödinger equation requires an initial value condition $\varphi(t=0)=\varphi_{0}$ that completely determines the dynamics of the system.

In this paper, we are especially concerned with a special form of $v(\mathbf{r}, t)$ that stems from time-dependent density functional theory (TDDFT). ${ }^{1,2}$ TDDFT can be viewed as a reformulation of time-dependent quantum mechanics where the basic variable is no longer the many-body wave function, but the time-dependent electron density $n(\mathbf{r}, t)$. The density can be obtained from the solution of a set of one-body equations, the so-called Kohn-Sham equations, that have the same form as Eq. (1). The potential that enters the Kohn-Sham equations is normally written as a sum of three terms,

$$
v_{\mathrm{KS}}(\mathbf{r}, t)=v_{\mathrm{ext}}(\mathbf{r}, t)+\int d^{3} r^{\prime} \frac{n\left(\mathbf{r}^{\prime}, t\right)}{\left|\mathbf{r}-\mathbf{r}^{\prime}\right|}+v_{\mathrm{xc}}[n](\mathbf{r}, t) .
$$

The first term is the external potential felt by the electrons, usually generated by some set of nuclei, and possibly by an external electromagnetic field. This term may also include an imaginary part describing absorbing boundary conditionsthe Hamiltonian in this case becomes non-Hermitian. This is important when descring open quantum systems as needed, for example, in molecular transport and photoemission 
processes. The next term, the Hartree potential, describes the classical part of the interaction between the electrons, while the exchange-correlation potential $v_{\mathrm{xc}}$ accounts for all other nontrivial many-body contributions. Note that both the Hartree and exchange-correlation potentials are functionals of the density and are therefore intrinsically time dependent.

Formally, the solution of Eq. (1) may be written as

$$
\varphi(t)=\hat{U}(t, 0) \varphi_{0}=\mathcal{T} \exp \left\{-i \int_{0}^{t} d \tau \hat{H}(\tau)\right\} \varphi_{0},
$$

where $\mathcal{T} \exp$, the time-ordered exponential, is a shorthand notation for

$$
\begin{aligned}
\hat{U}(t, 0)= & \sum_{n=0}^{\infty} \frac{(-i)^{n}}{n !} \int_{0}^{t} d \tau_{1} \int_{0}^{t} d \tau_{2} \cdots \int_{0}^{t} d \tau_{n} \\
& \times \mathcal{T}\left\{\hat{H}\left(\tau_{1}\right) \hat{H}\left(\tau_{2}\right) \cdots \hat{H}\left(\tau_{n}\right)\right\} .
\end{aligned}
$$

Equation (5) is an exact reformulation of the Schrödinger equation (see, for example, Ref. 3, Chap. 3). If the Hamiltonian commutes with itself at different times, we can drop the time ordering product; Moreover, if the Hamiltonian is time independent, the solution is simply

$$
\varphi(t)=\exp \{-i t \hat{H}\} \varphi_{0} .
$$

Unfortunately, this is not the case relevant for TDDFT, in any case, and in particular when applied to the description of the electronic dynamics under time-dependent external perturbations (as electric and magnetic fields, pulsed lasers, currents, particle scattering, etc.).

In practice, it is normally not convenient to obtain $\varphi(t)$ directly from $\varphi_{0}$ for a long interval $[0, t]$. Instead, one breaks $[0, t]$ into smaller time intervals, and, by making use of the well-known property $\hat{U}\left(t_{1}, t_{2}\right)=\hat{U}\left(t_{1}, t_{3}\right) \hat{U}\left(t_{3}, t_{2}\right)$, the full unitary time propagator is written as

$$
\hat{U}(t, 0)=\prod_{i=0}^{N-1} \hat{U}\left(t_{i}+\Delta t_{i}, t_{i}\right),
$$

where $t_{0}=0, t_{i+1}=t_{i}+\Delta t_{i}$, and $t_{N}=t$. In all cases studied in this paper we used a constant time step, i.e., $\Delta t_{i}=\Delta t$. However, it is possible to use variable time-step methods, especially if the algorithm implemented is able to choose optimally the time step to enhance the efficiency without compromising the accuracy. In any case, we deal with the problem of performing the short-time propagation,

$$
\begin{aligned}
\varphi(t+\Delta t) & =\hat{U}(t+\Delta t, t) \varphi(t) \\
& =\mathcal{T} \exp \left\{-i \int_{t}^{t+\Delta t} d \tau \hat{H}(\tau)\right\} \varphi(t) .
\end{aligned}
$$

Technically, the purpose of dividing $[0, t]$ into smaller intervals is twofold: the time dependence of $\hat{H}$ is alleviated and the norm of the exponential argument is reduced (the norm increases linearly with $\Delta t$ ). On top of these convenience reasons, there is a natural limit to the maximum size of $\Delta t$ : If $\omega_{\max }$ is the maximum frequency that we want to discern, $\Delta t$ cannot be larger than $\approx 1 / \omega_{\max }$. Below this $\Delta t_{\max }$, we are free to choose $\Delta t$ considering performance reasons. If $p(\Delta t)$ is the cost of propagating $\Delta t$ for a given method, one should then choose the $\Delta t$ that minimizes $p(\Delta t) / \Delta t$, the cost of propagating the wave function per unit time. The optimal cost number of a given method is $p\left(\Delta t_{\text {opt }}\right) / \Delta t_{\text {opt }}$, so the method that minimizes this optimal cost number can be viewed as the "best" method.

The value of $\omega_{\max }$ is either determined by the energy spectrum of the ground-state many-body Hamiltonian or by the frequency of the applied electromagnetic field. In the former case, the maximum frequency of the Hamiltonian is typically determined by the kinetic term. If the wave functions are expanded in a plane-wave representation, $\omega_{\max }$ is related to the maximum reciprocal lattice vector used in the expansion $\mathbf{G}_{\max }$; on the other hand, if we choose to work with a real-space discretization of the Hamiltonian, $\omega_{\max }$ is determined by the mesh spacing $h$. We have therefore

$$
\omega_{\max }=\frac{G_{\max }^{2}}{2}=\frac{2 \pi^{2}}{h^{2}} .
$$

In many cases the evolution will not probe the very high frequencies, so we can choose $\Delta t$ to be larger than $1 / \omega_{\max }$.

For a Hermitian Hamiltonian, the evolution operator is unitary, i.e.,

$$
\hat{U}^{\dagger}(t+\Delta t, t)=\hat{U}^{-1}(t+\Delta t, t) .
$$

This mathematical property is linked to the conservation of probability of the wave packet. Any desirable approximate propagator should be unitary, at least approximately, for Hermitian Hamiltonians. Another important property fulfilled by the exact evolution propagator is time-reversal symmetry,

$$
\hat{U}(t+\Delta t, t)=\hat{U}^{-1}(t, t+\Delta t) .
$$

(This property does not hold if a magnetic field is present; it must not be enforced if one wants to handle magnetic cases. However, any desirable algorithm should respect this property in the particular case where no magnetic field is applied.)

From a numerical point of view, the algorithm used to perform the time propagation should be "stable" and "accurate." The term stable is frequently used in a rather loose form. It is, however, possible to give it a precise definition: A propagator is stable below $\Delta t_{\max }$ if, for any $\Delta t<\Delta t_{\max }$ and $n>0, \hat{U}^{n}(t+\Delta t, t)$ is uniformly bounded. One way to assure that the algorithm is stable is by making it "contractive," which means that $\|\hat{U}(t+\Delta t)\| \leqslant 1$. Of course, if the algorithm is unitary, it is also contractive and hence stable; but if the algorithm is only approximatively unitary, it is better if it is contractive. The reason for this is easy to understand: the error is typically proportional to the norm. A contractive algorithm will reduce the norm, and in consequence the error will grow smaller; on the contrary, a noncontractive scheme will yield larger errors at each time step. The adverb "unconditionally" is sometimes added to these concepts to refer to algorithms that possess a given property independently of $\Delta t$ and of the spectral characteristics of $\hat{H}$ (e.g., unconditionally stable, etc.).

Although we are interested in solving the timedependent Kohn-Sham equations, many similar problems arise in various areas of science. It is not surprising, there- 
fore, that much work has been devoted in the past to the construction of approximations to time propagators. Most of the literature refers to nuclear wave-packet propagation, either in a quantum, semiclassical, or mixed schemes. The equations are, nevertheless, identical, and experience from this field may be translated to others. We learned from Kosloff's review, ${ }^{4}$ from the work of Lubich and co-workers, ${ }^{5-7}$ from the comparisons of Truong and others, ${ }^{8}$ and from other references that will be cited when appropriate. For the particular problem of TDDFT, we would like to mention the recent work of Sugino and Miyamoto. ${ }^{9}$ It is important to mention here the advances in the simulation of (adiabatic) molecular dynamics following the Car-Parrinello approach. ${ }^{10}$ The time integration is effectively performed using modified Verlet and Gaussian dynamics including multiple-time-scale methodologies. ${ }^{11}$ However, those works do not address the real electron dynamics of a system but a fictitious one determined by an effective electron mass and need to impose the orthogonality constrain for the wave functions (that is automatically fulfilled in the unitary propagation schemes to be described below). Of course, those techniques are going to be relevant here when addressing the combined electron/ion dynamics of the system under an external time perturbation.

We have implemented some of the most common approaches to the propagation of a quantum wave packet in our computer code OCTOPUS, ${ }^{12}$ a general purpose pseudopotential, real-space code. (By real space, or direct space, we mean that all functions are discretized in a grid, and that the Laplacian is approximated by finite differences. ${ }^{13}$ ) In this paper we review these approaches, and we provide several benchmarks in order to assert their efficiency in real-world applications. For some reviews on applications of TDDFT to complex nanostructures, biomolecules, and solids see Refs. 1,2 , and 14. General routines are available from осторUS website and can be used in more general contexts than the one discussed here.

Our discussion is separated into two parts: First we look at several algorithms to approximate $\exp (\hat{A})$, where $\hat{A}$ is a time-independent operator. In particular, polynomial expansions, projection in Krylov subspaces, and split-operator methods are investigated. We then discuss different approximations for the time-evolution operator, such as the midpoint and implicit rules, and Magnus expansions. Split-operator techniques can also be modified to approximate the full time dependent propagator. Note that, as the Hamiltonian is timedependent, the problem of approximating $\exp (\hat{A})$ is not equivalent to the problem of approximating the time propagator. However, the approximate propagators use exponentials of the form $\exp (\hat{A})$ as building blocks, where $\hat{A}$ typically has the form $-i \Delta t \hat{H}(\tau)$, for a given $\tau$.

\section{APPROXIMATIONS TO THE EXPONENTIAL OF AN OPERATOR}

In principle, the most desirable algorithm to calculate $\exp (\hat{A}) v$, where $v$ is an arbitrary vector, would begin with the evaluation of $\exp (\hat{A})$. In this way, we would be able to easily apply the exponential of the matrix $\hat{A}$ to any arbitrary vector.
Unfortunately, the methods that exist to calculate the exponential of a matrix are computationally limited to matrices of order less than a few thousands (for a recent review, please see Ref. 15). In a typical plane-wave or real-space calculation the Hamiltonian matrix can be of the order $\approx 2 \times 10^{5}$, and therefore way too large to apply any of these methods. In fact, the size of the Hamiltonian does not even permit its full storage in matrix form. A similar situation appears when solving the linear system $\hat{A} x=v$ : The evaluation of $\hat{A}^{-1}$ would allow the solution of the linear system for any vector $v$. However, the effort to invert the matrix $\hat{A}$ grows as $N^{3}$, where $N$ is the dimension of the matrix.

The alternative is to use iterative methods that yield directly $\exp (\hat{A}) v$ for a particular choice of the vector $v$. These methods have a much better scaling with the order of the matrix. In this paper we focus on three different techniques: polynomial expansion of the exponential, either in the standard base or in the Chebyshev base, splitting schemes, and Krylov subspace projection techniques. In the following we present a brief description of these methods followed by some numerical results illustrating their relative performance. To simplify our presentation we assume, without loss of generality, $\hat{A}=-i \hat{H} \Delta t$.

\section{A. Polynomial expansions}

The exponential of a matrix $\hat{A}$ is defined by the Taylor expansion

$$
\exp (\hat{A})=\sum_{n=0}^{\infty} \frac{1}{n !} \hat{A}^{n}
$$

This suggests an obvious method to approximate the exponential:

$$
\operatorname{taylor}_{k}\{\hat{A}, v\}=\sum_{n=0}^{k} \frac{1}{n !} \hat{A}^{n} v
$$

For a given $k$, the method is of order $k$ and requires $k$ matrix-vector operations. It amounts to expanding the exponential function in the standard base of polynomials, $\left\{1, x, x^{2}, \ldots\right\}$. The truncation of the infinite series at a given $k$ breaks the unitarity of the exponential. It turns out that $k=4$ is particularly suited for our applications; ${ }^{16} k=2$ is unconditionally unstable; $k=4$ is conditionally stable; $k=6$ is also conditionally stable but for smaller values of $\Delta t$. This fourth order expansion was already used in Ref. 17 for the description of the optical response of clusters computed by a time-propagation scheme and in later applications of the OCTOPUS code. ${ }^{2,14}$

The standard base of polynomials is not the only choice; one can use any given (complete and orthonormal) base $\left\{P_{n}(x)\right\}_{n=0}^{\infty}$. It is well known that Chebychev polynomial approximations are optimal for approximating functions, so we define

$$
\operatorname{cheb}_{k}\{\hat{A}, v\}=\sum_{n=0}^{k} c_{n} T_{n}(\hat{A}) v,
$$


where $T_{n}$ is the Chebychev polynomial of order $n$. For a skew-Hermitian matrix $\hat{A}$ of the form $-i \hat{H} \Delta t$ the Chebyshev expansion reduces to ${ }^{18}$

$$
\operatorname{cheb}_{k}\{-i \hat{H} \Delta t, v\}=\sum_{n=0}^{k}\left(2-\delta_{n 0}\right)(-i)^{n} J_{n}(\Delta t) T_{n}(\hat{H}) v,
$$

where $J_{n}$ are the Bessel functions. The resulting method is also of order $k$, and, thanks to Clenshaw's algorithm, ${ }^{19}$ requires $k$ matrix-vector operations. As the Chebychev polynomials are only defined in the range $[-1,1]$, the Hamiltonian has to be scaled so that its spectrum lies within this range before using Eq. (15). The application of Chebychev polynomials to chemistry was pioneered by Kosloff; ${ }^{4}$ more recent studies can be found in Refs. 20 and 21.

\section{B. Krylov subspace projection}

The $m$ th Krylov subspace, $\mathcal{K}_{m}\{\hat{A}, v\}$, for a given operator $\hat{A}$ and vector $v$ is defined as

$$
\mathcal{K}_{m}\{\hat{A}, v\}=\operatorname{span}\left\{v, \hat{A} v, \hat{A}^{2} v, \ldots, \hat{A}^{m-1} v\right\} .
$$

Note that $\operatorname{dim} \mathcal{K}_{m}\{\hat{A}, v\}$ may be smaller than $m$ if $v$ does not have non-null components of at least $m$ distinct eigenvectors of $\hat{A}$. The Lanczos procedure generates recursively an orthonormal base $\left\{v_{i}\right\}_{i=1}^{m}$ such that

$$
\hat{A} \hat{V}_{m}=\hat{V}_{m} \hat{H}_{m}+h_{m+1, m} v_{m+1} e_{m}^{T},
$$

where $\hat{V}_{m}=\left[v_{1}, \ldots, v_{m}\right], \hat{H}_{m}$ is an $m \times m$ symmetric tridiagonal matrix (upper Heisenberg if $\hat{H}$ is non-Hermitian), and $e_{i}$ is the $i$ th unit vector in $\mathrm{C}^{m} . \hat{H}_{m}$ is the projection of $\hat{A}$ onto $K_{m}\{\hat{A}, v\}$ and is the upper-left part of $\hat{H}_{m+1}$. By induction, it can be proved $^{22}$ that for any polynomial $p_{m-1}$ of degree $\leqslant m-1$

$$
p_{m-1}(\hat{A}) v=\hat{V}_{m} p_{m-1}\left(\hat{H}_{m}\right) \hat{V}_{m}^{T} v=\hat{V}_{m} p_{m-1}\left(\hat{H}_{m}\right) e_{1} .
$$

This suggests a method to approximate any function, and specifically the exponential,

$$
\operatorname{lanczos}_{k}\{\hat{A}, v\}=\hat{V}_{k} \exp \left(\hat{H}_{k}\right) e_{1} .
$$

Very good approximations are often obtained for relatively small $k$. The calculation of $\exp \left(\hat{H}_{k}\right)$ can be computed by any of the methods described in Ref. 15. The Krylov subspace projection is an order $k$ method that requires $k$ matrix-vector operations. To within our knowledge, it was Park and Light ${ }^{23}$ who first applied the Lanczos algorithm to chemistry; Hochbruck and Lubich ${ }^{24}$ made a thorough mathematical analysis of the technique.

\section{Splitting techniques}

The split-operator (SO) technique takes advantage of the fact that the Hamiltonian is composed of two terms, one diagonal in Fourier space-the kinetic operator $\hat{T}$ - and the other diagonal (or almost diagonal) in real space - the potential operator $\hat{V}$. The idea is to approximate the propagator by the following product of exponentials:

$$
\begin{aligned}
\operatorname{split}\{-i \Delta t \hat{H}, v\}= & S_{2}(-i \Delta t \hat{H}) v=\exp \left\{-i \frac{\Delta t}{2} \hat{T}\right\} \\
& \times \exp \{-i \Delta t \hat{V}\} \exp \left\{-i \frac{\Delta t}{2} \hat{T}\right\} v .
\end{aligned}
$$

This decomposition neglects terms involving the commutator $[\hat{T}, \hat{V}]$ and higher order commutators, and is of $\mathcal{O}\left(\Delta t^{2}\right)$. Equation (20) is sometimes called "potential referenced split operator," since the potential term appears sandwiched between the two kinetic terms. A "kinetic referenced" scheme is equally legitimate. Since the three exponentials may be computed exactly, it is always unitary and unconditionally stable, providing a very reliable second-order method. The split operator was first introduced in physics or chemistry by Feit and co-workers. ${ }^{25,26}$

Besides the simple SO method, a wide variety of other splitting schemes have been proposed and studied. ${ }^{27-29}$ One of these, the fourth-order symmetric decomposition, was studied and applied to TDDFT by Sugino and Miyamoto, ${ }^{9}$

$$
\operatorname{suzuki}\{-i \Delta t \hat{H}, v\}=\prod_{j=1}^{5} S_{2}\left(-i p_{j} \Delta t \hat{H}\right) v,
$$

where the $p_{j}$ are a properly chosen set of real numbers. Hereon we will call this scheme "Suzuki-Trotter" (ST), following the nomenclature of Ref. 9.

\section{Performance comparisons}

We start by comparing the performance of the fourthorder Taylor [Eq. (13)] and Chebyshev [Eq. (14)] expansions, and of the Lanczos method [Eq. (19)]. The cost of these three methods is roughly proportional to the number of $\hat{H} \varphi$ operations. Our test consists in applying $\exp \left\{-i \Delta t \hat{H}_{K S}\right\}$ to the $1 s$ orbital of sodium, to one $2 p$ orbital of carbon, and to one $5 d$ orbital of the gold atom. The orbitals are slightly perturbed from their ground-state shape, and $\hat{H}_{K S}$ is the ground state Kohn-Sham Hamiltonian (we are therefore ignoring the time dependence of the Hamiltonian). We define $p(\Delta t)$ to be the number of $\hat{H} \varphi$ operations necessary to achieve a given accuracy (defined as the norm of the difference between the exact solution vector and the approximate one). The function $p(\Delta t)$ measures the computational cost in units of the cost of a matrix-vector operation. Dividing by $\Delta t$ we obtain the computational cost of propagating the state per unit time, $p(\Delta t) / \Delta t$. Our results are presented in Fig. 1.

For small values of $\Delta t$, Lanczos and the Taylor expansion behave similarly, whereas the Cheby-chev expansion is slightly worse. As we increase $\Delta t$, the efficiency improves for all methods, reaching an optimal value for the Taylor expansion, after which increasing $\Delta t$ increases $p(\Delta t) / \Delta t$. The curves obtained with the Chebychev expansion also reach a minimum, although much less pronounced. The Lanczos method, on the other hand, becomes more efficient as we increase $\Delta t$. We should recall that the maximum value of $\Delta t$ is determined not by the propagation method but by the time dependence of the Hamiltonian. Below $\Delta t_{\max }$ the value of $\Delta t$ should be chosen in order to minimize $[p(\Delta t) / \Delta t]$. From our results it is clear that, for moderate to 


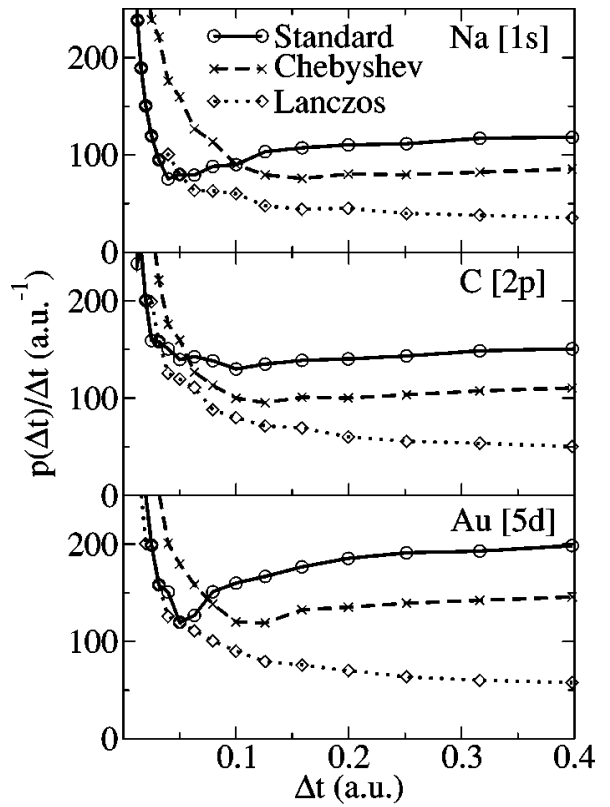

FIG. 1. Number of Hamiltonian-wave-function operations per unit time, as a function of $\Delta t$ for the Taylor (solid) and Chebyshev (dashed) expansions, and for the Lanczos projection method (dotted)

large values of $\Delta t$, the best choice is the Lanczos method, followed by the Chebychev expansion. For smaller $\Delta t$, either the Taylor expansion or Lanczos should be used.

The Lanczos method has an additional noteworthy advantage over the other methods: There is a simple and reliable criterion to estimate the error in approximating the propagator by $\operatorname{lanczos}_{k}\{-i \hat{H} \Delta t, v\}:{ }^{6}$ Using the same notation as in Sec. II B, the magnitude

$$
h_{m+1, m}\left|\left[\exp \left(\hat{H}_{m}\right)\right]_{m, 1}\right|
$$

is an estimator for the error made by the $m$ th order expansion $\left(\left[\exp \left(\hat{H}_{m}\right)\right]_{m, 1}\right.$ is the $(m, 1)$ entry of the $m$-dimensional matrix $\left.\exp \left(\hat{H}_{m}\right)\right)$. If this error estimator is larger than a prescribed tolerance $\varepsilon$, we can (i) for a fixed $\Delta t$, increase the dimension of the Krylov subspace $k$ until the precision criterion is met, or (ii) for a fixed $k$, decrease $\Delta t$-the error behaves as $\mathcal{O}\left(\Delta t^{m+1}\right)$, for $\Delta t$ small enough. The implementation of the variable time-step method (ii) is complicated if several orbitals have to be propagated at the same time, as the optimum $\Delta t$ might not be the same for every orbital. Therefore, we have relied on method (i).

We would like to mention that we tried a few other possible improvements of the Lanczos method without success. The concept of Krylov subspace and the Lanczos recursion may be generalized to obtain the banded Lanczos algorithms, where the initial vector $v$ is extended to a set of $k$ vectors: $K_{m}\left(A, v_{1}, \ldots, v_{k}\right)=\operatorname{span}\left\{A^{(l)} v_{j}\right\}_{j=1, \ldots, k}^{l=0, \ldots, 1}$. Banded Lanczos algorithms are used successfully for diagonalizing large sparse operators (such as the Kohn-Sham Hamiltonian written in real space). We used banded Lanczos algorithms within two different approaches: (i) supplementing the orbital we want to propagate, $v_{1}=\varphi(t)$, with an approximation to the solution $\exp \{-i \hat{H} \Delta t\} v$ obtained from the previous iteration, $v_{2}=\operatorname{lanczos}\{-i \hat{H} 2 \Delta t, \varphi(t-\Delta t)\}$; and (ii) propagat-

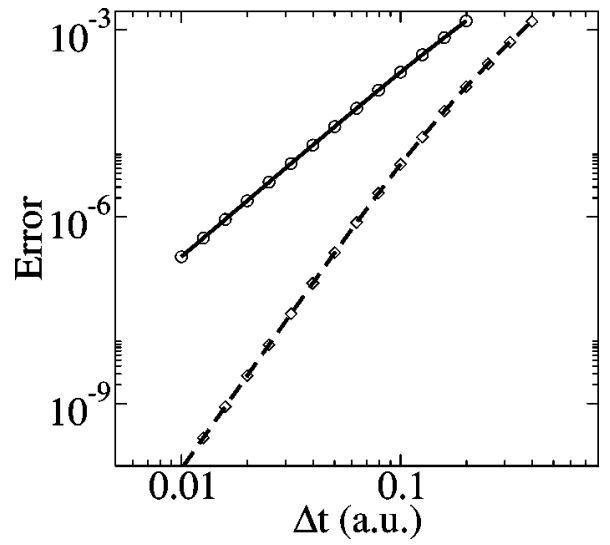

FIG. 2. Comparison of the second-order split operator (SO, solid) and the fourth-order Suzuki-Trotter (ST, dashed) schemes.

ing at the same time a given number of Kohn-Sham orbitals with the banded Lanczos algorithm. Unfortunately, neither of these two methods reduced the cost of the original algorithm. (The failure for our particular problem and computational implementation does not imply, of course, that they could be advantageously employed under different conditions.)

In Fig. 2 we compare the second-order split-operator scheme to the fourth-order Suzuki-Trotter propagator. The plot depicts the error in the propagation versus $\Delta t$ for the $5 d$ orbital of Au referred above. The order 2 and order 4 behavior of the schemes is clearly demonstrated by the respective slopes of the curves. For a given $\Delta t$, the error yielded by the higher order technique is always lower. However, the computational cost of the Suzuki-Trotter scheme is five times larger than that of the simple split operator. For a given accuracy, the higher order scheme will be more profitable than the normal split operator if the time step $\Delta t$ is more than five times smaller. In our experience, that is not always the case, and depends on the system under consideration and on the level of accuracy seeked. Sugino and Miyamoto ${ }^{9}$ report the superiority of the higher order method for their implementation and cases studied.

Now we compare the splitting techniques to the best of the polynomial expansions, i.e., the Lanczos method. As these are very different approaches, it is hard to find an appropriate cost function to compare them. We have therefore defined a "quality" number based on the computer time necessary to achieve a given accuracy. The procedure to achieve the desired accuracy is different in both approaches; for the Lanczos method we fix $\Delta t=0.2$ a.u. and increase the dimension of the Krylov subspace as needed; for the splitting techniques we decrease $\Delta t$ until fulfilling the accuracy requirement. We then divide the total propagation time by the time necessary to perform $\hat{H} \varphi$ and by $\Delta t$ to obtain the cost function per unit time $p(\Delta t) / \Delta t$. (This procedure is consistent with our previous definition.) In Table I we show the cost function of the splitting techniques and of the Lanczos method for several test cases. The Lanczos scheme is clearly superior in all cases. Between the two splitting schemes we find that the higher order expansion does not improve the performance of the standard split operator. 
TABLE I. Performance number, $p(t) / \Delta t$, for the split-operator (SO), higher-order Suzuki-Trotter (ST), and Lanczos subspace methods.

\begin{tabular}{cccc}
\hline \hline Method & $\mathrm{Na}[1 s]$ & $\mathrm{C}[2 p]$ & $\mathrm{Au}[5 d]$ \\
\hline Lanczos & 48 & 63 & 75 \\
SO & 184 & 192 & 202 \\
ST & 227 & 231 & 244 \\
\hline \hline
\end{tabular}

\section{ANALYSIS OF INTEGRATORS FOR THE TDSE}

We now turn to the problem of approximating the evolution operator $\hat{U}(t+\Delta t, t)$, i.e., finding an approximation for $\varphi(t+\Delta t)$ from the knowledge of $\varphi(\tau)$ and $\hat{H}(\tau)$ for 0 $\leqslant \tau \leqslant t$. Most methods also require the knowledge of the Hamiltonian at some points in time between $t \leqslant \tau \leqslant t+\Delta t$. To obtain this quantity, one can, e.g., extrapolate the Hamiltonian using a polynomial fit to $n$ previous steps. However, this can reduce the accuracy of the propagator. To be fully consistent the following method can be employed: (i) obtain $\hat{H}(\tau)$ through extrapolation; (ii) propagate $\varphi$ to get $\varphi(t$ $+\Delta t$ ); (iii) from $\varphi(t+\Delta t)$ calculate $\hat{H}(t+\Delta t)$; (iv) obtain $\hat{H}(\tau)$ by interpolating between $\hat{H}(t)$ and $\hat{H}(t+\Delta t)$; (v) repeat steps (ii)-(iv) until self-consistency is reached. For small time steps, the step (i) may be sufficient.

In the following, we briefly describe several propagators that we have investigated within the framework of real-space TDDFT calculations. For the theoretical description of the properties of the propagators (unitarity, time reversibility), we assume that $\hat{H}(\tau)$ is properly obtained using the abovementioned self-consistent procedure, and that all numerical operations (calculation of the exponential of an operator, solution of a linear system, etc.) are performed exactly. We then present our results concerning the performance of the methods.

\section{A. Implicit midpoint rule}

The implicit midpoint rule, also known as CrankNicholson $(\mathrm{CN})$ method, is a member of the family of methods sometimes referred to as "classical propagators," a family in which we may include Euler's method, implicit or explicit Runge-Kutta, multistep algorithms, etc. These propagators are of "general purpose," and have well-known numerical properties. However, the typical form of the Hamiltonian matrix, given by Eq. (2), has traditionally favored the use of splitting techniques or other methods. We mention the $\mathrm{CN}$ method mainly for historical reasons, as our experience and that of others ${ }^{5}$ advice the use of different techniques.

The implicit midpoint rule is defined by

$$
\hat{U}_{\mathrm{CN}}(t+\Delta t, t)=\frac{1-\frac{i}{2} \Delta t \hat{H}(t+\Delta t / 2)}{1+\frac{i}{2} \Delta t \hat{H}(t+\Delta t / 2)} .
$$

The problem of propagating an orbital with this scheme is usually cast in the solution of the linear system:

$$
\hat{L} \varphi(t+\Delta t)=b,
$$

where $\hat{L}=\hat{I}+i(\Delta t / 2) \hat{H}(t+\Delta t / 2)$ and $b=[\hat{I}-i(\Delta t / 2) \hat{H}(t$ $+\Delta t / 2)] \varphi(t)$. The $\mathrm{CN}$ scheme is unitary and preserves timereversal symmetry.

\section{B. Exponential midpoint rule}

The exponential midpoint (EM) rule consists in approximating the propagator by the exponential calculated at time $t+\Delta t / 2$,

$$
\hat{U}_{\mathrm{EM}}(t+\Delta t, t) \equiv \exp \{-i \Delta t \hat{H}(t+\Delta t / 2)\} .
$$

The actual propagation can then be done by any of the methods described in Sec. II. If we assume that the exponential is calculated exactly and that $\hat{H}(t+\Delta t / 2)$ is obtained selfconsistently then this method is also unitary and time reversible. In practice this method requires small time steps to be stable.

\section{Time-reversal symmetry based propagator}

In a time-reversible method, propagating backwards $\Delta t / 2$ starting from $\varphi(t+\Delta t)$ or propagating forwards $\Delta t / 2$ starting from $\varphi(t)$ should lead to the same result. By using the simplest approximation to the propagator, this statement leads to the condition

$\exp \left\{+i \frac{\Delta t}{2} \hat{H}(t+\Delta t)\right\} \varphi(t+\Delta t)=\exp \left\{-i \frac{\Delta t}{2} \hat{H}(t)\right\} \varphi(t)$.

Rearranging the terms, we arrive at an approximation to the propagator

$$
\begin{aligned}
\hat{U}_{\mathrm{ETRS}}(t+\Delta t, t)= & \exp \left\{-i \frac{\Delta t}{2} \hat{H}(t+\Delta t)\right\} \\
& \times \exp \left\{-i \frac{\Delta t}{2} \hat{H}(t)\right\} .
\end{aligned}
$$

We call this method the enforced time-reversal symmetry (ETRS) method.

\section{Splitting techniques}

The splitting techniques have been described in Sec. II C as a way to approximate the exponential of a timeindependent Hamiltonian. By combining them, e.g., with the above mentioned EM or ETRS method one obtains an approximation for the full propagator based on either the splitoperator or on the Suzuki-Trotter scheme. There is, however, an alternative way to improve the splitting schemes with small added computational cost.

Watanabe and Tsukada ${ }^{30}$ have recently combined the EM approximation with the split-operator method. In practice, this consists in setting $\hat{V}=\hat{V}(t+\Delta t / 2)$ in Eq. (20). If this potential is obtained accurately we end up with an order 2 method, otherwise the method is of first order. There is, however, a simpler alternative: 


$$
\begin{aligned}
\hat{U}_{\mathrm{SO}}(t+\Delta t, t)= & S_{2}\left[-i \Delta t\left(\hat{T}+\hat{V}^{\prime}\right)\right]=\exp \left\{-\frac{1}{2} i \Delta t \hat{T}\right\} \\
& \times \exp \left\{-i \Delta t \hat{V}^{\prime}\right\} \exp \left\{-\frac{1}{2} i \Delta t \hat{T}\right\}
\end{aligned}
$$

where the potential operator $\hat{V}^{\prime}$ is defined by

$$
\hat{V}^{\prime}=v_{\mathrm{ext}}(\mathbf{r}, t+\Delta t / 2)+\int d^{3} r^{\prime} \frac{n^{\prime}\left(\mathbf{r}^{\prime}\right)}{\left|\mathbf{r}-\mathbf{r}^{\prime}\right|}+v_{\mathrm{xc}}\left[n^{\prime}\right](\mathbf{r}, t) .
$$

In this expression $n^{\prime}$ is the density built after applying the first kinetic exponential in Eq. (28). In other words, the modified SO method is the following: (i) apply the first kinetic term; (ii) recalculate the density and obtain the KohnSham potential, and (iii) apply the potential term and the second kinetic term. In this simple way we recover an order 2 method.

For the higher order Suzuki-Trotter technique, Suzuki provided a time-dependent version:

$$
\hat{U}_{\mathrm{ST}}(t+\Delta t, t)=\prod_{j=1}^{5} S_{2}\left[-i p_{j} \Delta t \hat{H}\left(t_{j}\right)\right],
$$

where the times $t_{j}$ are related to the set $p_{j}$ trough $t_{j}=t$ $+\left(p_{1}+\cdots+p_{j} / 2\right) \Delta t$. Once again, the potential between $t$ and $t+\Delta t$ has to be properly extrapolated to obtain a true order 4 technique (similar for higher order expansions).

\section{E. Magnus expansions}

As noted previously, $\hat{U}(t+\Delta t, t)$ does not reduce to a simple exponential of the form $\exp \{-i \Delta t \hat{H}(t)\}$ unless the Hamiltonian is time independent. One may ask if there exists an operator $\hat{\Omega}(t+\Delta t, t)$ such that $\hat{U}(t+\Delta t, t)=\exp \{\hat{\Omega}(t$ $+\Delta t, t)\}$. Magnus ${ }^{31}$ answered this question positively in 1954: there exists an infinite series, convergent at least for some local environment of $t$, such that

$$
\hat{\Omega}(t+\Delta t, t)=\sum_{k=1}^{\infty} \hat{\Omega}_{k}(t+\Delta t, t)
$$

There also exists a procedure to generate the exact $\hat{\Omega}_{k}$ operators: ${ }^{32}$

$$
\hat{\Omega}_{k}(t+\Delta t, t)=\sum_{j=0}^{k-1} \frac{B_{j}}{j !} \int_{t}^{t+\Delta t} \hat{S}_{k}^{j}(\tau) d \tau,
$$

where $B_{j}$ are Bernoulli numbers and the operators $S$ are recursively generated

$$
\begin{aligned}
& \hat{S}_{1}^{0}(\tau)=-i \hat{H}(\tau) ; \hat{S}_{k}^{0}(\tau)=0 \quad(k>1), \\
& \hat{S}_{k}^{j}(\tau)=\sum_{m=1}^{k-j}\left[\hat{\Omega}_{m}(t+\Delta t, t), \hat{S}_{k-m}^{j-1}(\tau)\right] \quad(1 \leqslant j \leqslant k-1) .
\end{aligned}
$$

For example, the first two terms of the recurrence are

$$
\hat{\Omega}_{1}(t+\Delta t, t)=\int_{t}^{t+\Delta t} d \tau_{1}\left[-i \hat{H}\left(\tau_{1}\right)\right],
$$

$$
\hat{\Omega}_{2}(t+\Delta t, t)=\int_{t}^{t+\Delta t} d \tau_{1} \int_{t}^{\tau_{1}} d \tau_{2}\left[-i \hat{H}\left(\tau_{1}\right),-i \hat{H}\left(\tau_{2}\right)\right] .
$$

In general, the $k$ th term will be a $k$-dimensional integral of a sum of commutators of $\hat{H}$ at different times. An approximation of order $2 n$ to the full Magnus operator (and hence, to the evolution operator) is achieved by truncating the Magnus series to $n$th order and approximating the integrals through an $n$th order quadrature formula. The exponential midpoint rule can be regarded as the second-order Magnus expansion, $\hat{U}_{\mathrm{EM}}=\hat{U}_{\mathrm{M}(2)}$, since

$$
\hat{\Omega}_{\mathrm{M}(2)}(t+\Delta t, t)=-i \hat{H}(t+\Delta t / 2) \Delta t .
$$

The fourth-order Magnus expression is constructed by taking the first two terms in the Magnus series and using, for example, a two-point Gaussian quadrature to approximate the integrals. The result is

$$
\begin{aligned}
\hat{\Omega}_{\mathrm{M}(4)}(t+\Delta t, t)= & -i \frac{\Delta t}{2}\left[\hat{H}\left(t_{1}\right)+\hat{H}\left(t_{2}\right)\right] \\
& -\frac{\sqrt{3} \Delta t^{2}}{12}\left[\hat{H}\left(t_{2}\right), \hat{H}\left(t_{1}\right)\right],
\end{aligned}
$$

where $t_{1,2}=t+[(1 / 2) \mp \sqrt{3} / 6] \Delta t$ are the Gauss quadrature sampling points. For the specific case of the Kohn-Sham Hamiltonian, the fourth-order Magnus propagator has the form

$$
\hat{U}_{\mathrm{M}(4)}(t+\Delta t, t)=\exp \left\{-i \Delta t \hat{H}_{\mathrm{M}(4)}(t, \Delta t)\right\},
$$

where the modified Hamiltonian $\hat{H}_{\mathrm{M}(4)}(t, \Delta t)$ operator is defined as

$$
\hat{H}_{\mathrm{M}(4)}(t, \Delta t)=\bar{H}(t, \Delta t)+i\left[\hat{T}+\hat{V}_{\mathrm{ext}}^{\mathrm{nonlocal}}, \overline{\Delta V}(t, \Delta t)\right],
$$

where only the nonlocal components of the Kohn-Sham Hamiltonian contribute to the commutator, and with the definitions

$$
\begin{aligned}
& \bar{H}(t, \Delta t)=\hat{T}+\frac{1}{2}\left\{\hat{V}_{\mathrm{KS}}\left(t_{1}\right)+\hat{V}_{\mathrm{KS}}\left(t_{2}\right)\right\}, \\
& \overline{\Delta V}(t, \Delta t)=\frac{\sqrt{3}}{12} \Delta t\left\{\hat{V}_{\mathrm{KS}}\left(t_{2}\right)-\hat{V}_{\mathrm{KS}}\left(t_{1}\right)\right\} .
\end{aligned}
$$

Expression (40) assumes that the nonlocal part of the KohnSham Hamiltonian does not vary significantly in the interval of interest $(t, t+\Delta t)$. This nonlocal component is part of the ionic pseudopotentials used in electronic structure calculations and in consequence its variation is associated to the ionic movement. In principle, this movement should be negligible in the electronic time scale that determines $\Delta t$, and this is the reason for that assumption.

The fourth-order Magnus expansion involves the computation of one commutator. The number of such commutators grows rapidly with increasing order, although some work has recently been devoted to significantly reduce this number. ${ }^{33}$ The Magnus expansion has received a great deal of attention from the chemistry and physics community. A very recent in-depth study of the scheme may be found in Ref. 34; The first application to the field of quantum molecular systems 


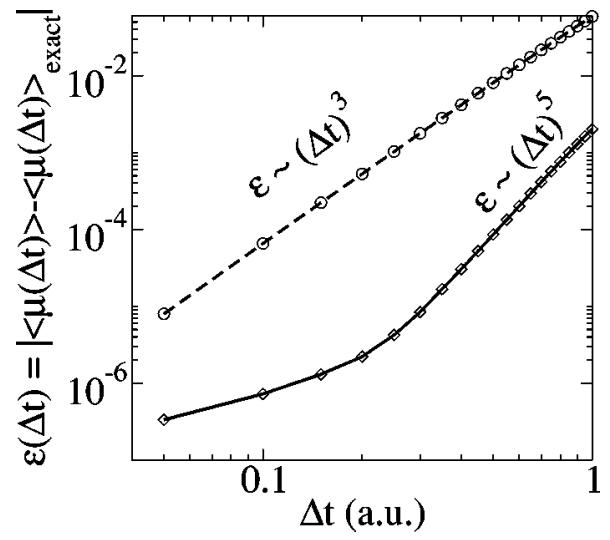

FIG. 3. Error in the dipole moment, for the exponential midpoint (dashed line) and fourth-order Magnus (solid) methods, as a function of $\Delta t$.

was made by Milfeld and Wyatt ${ }^{35}$ in 1983 . However, we could not find any application of the Magnus expansions in the field of electronic structure calculations.

\section{F. Performance comparisons}

In this section we compare the different approximations to the time-evolution operator. We have analyzed the performance of the EM, ETRS, splitting techniques, and Magnus integrators. It turns out that, in general, the EM and ETRS techniques have very similar behaviors: The time step required for the EM propagation is roughly half the time step for the ETRS scheme, but the cost of each propagation step is also halved (the EM scheme requires the evaluation of one exponential, while the ETRS requires two). It is also quite clear already from Table I that the splitting techniques are not adequate for this kind of problems.

We are left with the comparison of the fourth-order Magnus expansion to the EM (which indeed is equivalent to a second-order Magnus expansion). In Fig. 3 we plot the error in the dipole moment, after performing a single step propagation, $\hat{U}(\Delta t, 0)$. The system under consideration is a $\mathrm{Na}_{8}$ cluster in the jellium model, excited from its ground state by a laser pulse of frequency 2 Hartree. The fourth-order method is, as expected, more precise. However, the cost of applying the Magnus operator, Eq. (40), is roughly 50\% larger than applying the usual Hamiltonian; $p_{\mathrm{M}(4)}(\Delta t)$ $\approx \frac{3}{2} p_{\mathrm{EM}}(\Delta t)$. Therefore, the use of the fourth-order Magnus expansion will be advantageous over the simpler EM method only if one can use time steps more than 1.5 times larger. Of course, the use of longer time steps may be hindered by the approximation used for the exponential (Lanczos, Chebychev, etc.).

Heuristically, the fourth-order Magnus expansion becomes useful when the Hamiltonian has very high frequencies, i.e., if the Hamiltonian is strongly time dependent. An example is shown in Fig. 4 where we plot the evolution of the dipole moment of the above-mentioned $\mathrm{Na}_{8}$ example during ten atomic units of time. The time step is one atomic unit. The $\mathrm{M}(4)$ scheme is clearly superior in this range of frequencies or, to be more precise, for this range of the product $\omega \delta t$, where $\omega$ is the highest frequency present in the evolution. It is able to "follow" the exact evolution of the dipole moment,

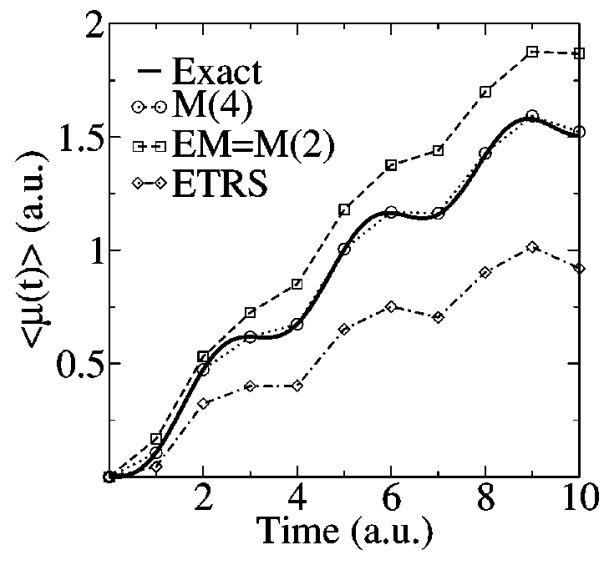

FIG. 4. Evolution of the dipole moment of $\mathrm{Na}_{8}$ (in the jellium model), subject to an intense laser field, calculated via the methods indicated in the legend.

whereas the lower-order methods deviate from it, eventually rendering the evolution meaningless. Note that the very highfrequency oscillations ( 2 Hartree $\approx 54 \mathrm{eV}$ ) shown in the figure correspond to the laser field, not to the internal states of the cluster. (The plasmon response of this kind of clusters lies typically within $2-3 \mathrm{eV}$.) On the other hand, the steady increase in the dipole moment, to which the laser frequency is superimposed, corresponds to the beginning of the oscillation of the plasmon. Of course, the dipole calculated with the EM method will reduce to the exact curve if we reduce $\Delta t$, but this makes the EM calculation much less favorable in computer time. For this particular example, it turns out that the use of the fourth-order Magnus expansion reduces the computational cost by a factor of 3 .

We stress again that the answer to the question EM versus $M(4)$ is problem dependent, and that the performance of both methods should be carefully compared before performing a long time evolution.

\section{CONCLUSIONS}

Unfortunately, we cannot name an "always optimal" algorithm for the propagation of the time-dependent KohnSham equations. The final choice depends on the internal characteristics of the physical system, and on the frequency and intensity of any existing external fields. Furthermore, the final performance of any method also depends on the specific implementation of the equations, and possibly also on the computer architecture. (For example, we have observed strong variations on the performance of the fast Fourier transforms, which have a definitive influence on the cost of the splitting techniques.) Nevertheless, and keeping in mind all these observations, we believe that the exponential midpoint rule combined with the Lanczos exponential approximator gives a very good algorithm to represent the time propagator for a wide range of systems. Moreover, if the problem involves high frequencies, it is also worth trying the higher order Magnus expansion. Although our numerical results were obtained using a TDDFT code based on real-space methods, we expect them to be fairly general and applicable to other implementations (e.g., plane waves). Furthermore, some of the knowledge may safely be transported to the numerical implementation of other theories where either 
Schrödinger-like propagators or methods to approximate the action of exponential of operators are sought-we dare to cite the diffusion Monte Carlo method, or the recently investigated idea of obtaining accurate electronic wave functions through exponentials of two-body operators, ${ }^{36}$ as examples where these findings may be useful.

\section{ACKNOWLEDGMENTS}

This work was supported by the European Community Research Training Network NANOPHASE (Grant No. HPRN-CT-2000-00167), Network of Excellence NANOQUANTA (Grant No. NOE 500198-2), Spanish MCyT (Grant No. MAT2001-0946) and the University of the Basque Country (Grant No. 9/UPV 00206.215-13639/2001). The computer time was granted by DIPC and CEPBA (Barcelona). We acknowledge fruitful discussions with G. F. Bertsch.

${ }^{1}$ E. Runge and E. K. U. Gross, Phys. Rev. Lett. 52, 997 (1984); E. K. U. Gross, J. Dobson, and M. Petersilka, in Density Functional Theory II, Topics in Current Chemistry Vol. 18, edited by R. F. Nalewajski (Springer, Berlin, 1986), p. 81; R. van Leeuwen, Int. J. Mod. Phys. B 15, 1969 (2001); K. Burke, M. Petersilka, and E. K. U. Gross, in Recent Advances in Density Functional Methods, edited by P. Fantucci and A. Bencini (World Scientific, Singapore, 2002), p. 67.

${ }^{2}$ A. Castro, M. A. L. Marques, J. A. Alonso, and A. Rubio, J. Comp. Theor. Nanoscience (in press); M. A. L. Marques and E. K. U. Gross, Annu. Rev. Phys. Chem. 55, 427 (2004).

${ }^{3}$ Fetter and Walecka, Quantum Theory of Many-Particle Systems (McGraw-Hill, New York, 1971).

${ }^{4}$ R. Kosloff, J. Phys. Chem. 92, 2087 (1988).

${ }^{5}$ C. Lubich, in Quantum Simulations of Complex Many-Body Systems: From Theory to Algorithms, edited by J. Grotendorst, D. Marx, and A. Muramatsu (John von Neumann Institute for Computing, Jülich, 2002), Vol. 10, p. 459.

${ }^{6}$ M. Hochbruck, C. Lubich, and H. Selhofer, SIAM J. Sci. Comput. (USA) 19, 1552 (1998).

${ }^{7}$ M. Hochbruck and C. Lubich, BIT 39, 620 (1999).
${ }^{8}$ T. N. Truong, J. J. Tanner, P. Bala, and J. A. McCammon, J. Chem. Phys. 96, 2077 (1992).

${ }^{9}$ O. Sugino and Y. Miyamoto, Phys. Rev. B 59, 2579 (1999); 66, 89901(E) (2002)

${ }^{10}$ R. Car and M. Parrinello, Phys. Rev. Lett. 55, 2471 (1985).

${ }^{11}$ M. R. Tuckerman and M. Parrinello, J. Chem. Phys. 101, 1302 (1994); 101, 1316 (1994).

${ }^{12}$ The octopus project is aimed at describing the electron-ion dynamics in finite and extended systems under the influence of time-dependent electromagnetic fields. The program can be freely downloaded from http:// www.tddft.org/programs/octopus. For details see M. A. L. Marques, A. Castro, G. F. Bertsch and A. Rubio, Comput. Phys. Commun. 151, 60 (2003).

${ }^{13}$ T. L. Beck, Rev. Mod. Phys. 72, 1041 (2000), and references therein.

${ }^{14}$ G. Onida, L. Reining, and A. Rubio, Rev. Mod. Phys. 74, 601 (2002).

${ }^{15}$ C. Moler and C. van Loan, SIAM Rev. 45, 3 (2003).

${ }^{16} \mathrm{~J}$. Giansiracusa (private communication).

${ }^{17}$ K. Yabana and G. F. Bertsch, Phys. Rev. B 54, 4484 (1996).

${ }^{18}$ H. Tal-Ezer and R. Kosloff, J. Chem. Phys. 81, 3967 (1984)

${ }^{19}$ C. W. Clenshaw, MTAC 9, 118 (1955).

${ }^{20}$ R. Baer and R. Gould, J. Chem. Phys. 114, 3385 (2001).

${ }^{21}$ R. Chen and H. Guo, Comput. Phys. Commun. 119, 19 (1999).

${ }^{22}$ Y. Saad, SIAM (Soc. Ind. Appl. Math.) J. Numer. Anal. 29, 209 (1992).

${ }^{23}$ T. J. Park and J. C. Light, J. Chem. Phys. 85, 5870 (1986).

${ }^{24}$ M. Hochbruck and C. Lubich, SIAM (Soc. Ind. Appl. Math.) J. Numer. Anal. 34, 1911 (1997).

${ }^{25}$ M. D. Feit, J. A. Fleck, and A. Steiger, J. Comput. Phys. 47, 412 (1982).

${ }^{26}$ M. D. Feit and J. A. Fleck, J. Chem. Phys. 78, 301 (1982).

${ }^{27}$ M. Suzuki, J. Phys. Soc. Jpn. 61, L3015 (1993).

${ }^{28}$ M. Suzuki and T. Yamauchi, J. Math. Phys. 34, 4892 (1992).

${ }^{29}$ T. Y. Mikhailova and V. I. Pupyshev, Phys. Lett. A 257, 1 (1999); A. D. Bandrauk and H. Shen, J. Chem. Phys. 99, 1185 (1993).

${ }^{30}$ N. Watanabe and M. Tsukada, Phys. Rev. E 65, 036705 (2002).

${ }^{31}$ W. Magnus, Pure Appl. Math. 7, 649 (1954).

${ }^{32}$ S. Klarsfeld and J. A. Oteo, Phys. Rev. A 39, 3270 (1989).

${ }^{33}$ S. Blanes, F. Casas, and J. Ros, BIT 40, 434 (2000).

${ }^{34}$ M. Hochbruck and C. Lubich, SIAM (Soc. Ind. Appl. Math.) J. Numer. Anal. 41, 945 (2003).

${ }^{35}$ K. F. Milfeld and R. E. Wyatt, Phys. Rev. A 27, 72 (1983).

${ }^{36}$ M. Nooijen, Phys. Rev. Lett. 84, 2108 (2000); H. Nakatsuji, J. Chem. Phys. 113, 2949 (2000); T. van Voorhis and M. Head-Gordon, ibid. 115, 5033 (2001); P. Piecuch, K. Kowalski, P. D. Fang, and K. Jedziniak, Phys. Rev. Lett. 90, 113001 (2003). 